\title{
Synergistic interaction of cigarette smoking and alcohol drinking with serum carotenoid concentrations: findings from a middle-aged Japanese population
}

\author{
Minoru Sugiura $^{1}$, Mieko Nakamura ${ }^{2}$, Kazunori Ogawa ${ }^{1}$, Yoshinori Ikoma ${ }^{1}$, Hikaru Matsumoto ${ }^{1}$, \\ Fujiko $\mathrm{Ando}^{3}$, Hiroshi Shimokata ${ }^{4}$ and Masamichi Yano ${ }^{1}$ \\ ${ }^{1}$ Research Team for Health Benefit of Fruit, National Institute of Fruit Tree Science, 485-6 Okitsunaka-cho, Shimizu-ku, Shizuoka \\ 424-0292, Japan \\ ${ }^{2}$ Department of Community Health and Preventive Medicine, Hamamatsu University School of Medicine, \\ 1-20-1 Handayama, Higashi-ku, Hamamatsu 431-3192, Japan \\ ${ }^{3}$ Department of Community Care Philanthropy, Aichi Shukutoku University, 23 Sakuragaoka, Chigusa-ku, \\ Nagoya 464-8671, Japan \\ ${ }^{4}$ Department of Epidemiology, National Institute for Longevity Sciences, 36-3 Gengo, Morioka-cho, Obu, \\ Aichi 474-8522, Japan \\ (Received 16 October 2008 - Revised 31 March 2009 - Accepted 1 April 2009 - First published online 19 May 2009)
}

\begin{abstract}
Previous studies have indicated low serum carotenoid concentrations among cigarette smokers and/or alcohol drinkers, but little is known about the interaction of smoking and drinking with serum carotenoids. We tested the hypothesis that smoking and drinking reduce serum carotenoid concentrations synergistically. A total of 1073 subjects (357 male and 716 female) who had received health examinations in the town of Mikkabi, Shizuoka Prefecture, Japan, participated in the study. The subjects were divided into six groups according to alcohol intake (non-drinkers, $<1 \mathrm{~g} / \mathrm{d}$; light drinkers, $\geq 1,<25 \mathrm{~g} / \mathrm{d}$; moderate-to-heavy drinkers, $\geq 25 \mathrm{~g} / \mathrm{d}$ ) and smoking status (non-smokers and current smokers). The dietary intakes and serum concentrations of six carotenoids (lycopene, $\alpha$-carotene, $\beta$-carotene, lutein, $\beta$-cryptoxanthin and zeaxanthin) within each group were evaluated cross-sectionally. The dietary intakes of all carotenoids did not differ in the six groups after adjusting for age and sex. The multivariate-adjusted means of the serum carotenoid concentrations in non-drinkers did not differ between non-smokers and current smokers. In contrast, the adjusted means of serum $\alpha$-carotene, $\beta$-carotene and $\beta$-cryptoxanthin were significantly lower than those with increased alcohol intake, and these lower serum carotenoids among alcohol drinkers were more evident in current smokers than in non-smokers. Serum lycopene of moderate-to-heavy drinkers was significantly lower than that of non-drinkers, but it was not influenced by smoking. Neither smoking nor drinking was associated with the serum concentrations of lutein and zeaxanthin. These results suggest that smoking and drinking may reduce the serum $\alpha$-carotene, $\beta$-carotene and $\beta$-cryptoxanthin concentrations in a synergistic manner.
\end{abstract}

Carotenoids: Smoking: Alcohol drinking: Cross-sectional studies

Antioxidant micronutrients, such as vitamins and carotenoids, exist in abundance in fruit and vegetables and have been known to contribute to the body's defence against reactive oxygen species ${ }^{(1,2)}$. Numerous epidemiological studies have demonstrated that a high dietary consumption of fruit and vegetables rich in carotenoids or with high serum carotenoid concentrations results in lower risks of certain cancers, diabetes and $\mathrm{CVD}^{(3-8)}$. These epidemiological studies have suggested that antioxidant carotenoids may have a protective effect against these diseases.

On the other hand, active smokers are exposed to reactive free radicals that are present in cigarette smoke ${ }^{(9,10)}$. Therefore, smoking is a potent oxidative stress in humans ${ }^{(11-13)}$. Furthermore, alcohol drinking also induces reactive oxygen species during its metabolism in the liver ${ }^{(14-16)}$. Oxidants and free radicals induced by cigarette smoking and/or alcohol drinking can cause damage to lipids, proteins, DNA, carbohydrates and other biomolecules ${ }^{(17,18)}$. In such circumstances, antioxidant micronutrients, such as carotenoids, may play important roles in defending against oxidative stress by efficiently quenching the production of singlet oxygen and free radicals. Numerous epidemiological studies have shown that serum carotenoid concentrations were low among cigarette smokers and alcohol drinkers ${ }^{(19-31)}$. However, there is limited information about the synergistic interaction of cigarette smoking and alcohol drinking with serum carotenoid concentrations. The major serum carotenoids are lycopene, $\alpha$-carotene, $\beta$-carotene, lutein, $\beta$-cryptoxanthin and zeaxanthin, and they account for more than $90 \%$ of the circulating carotenoids in humans ${ }^{(32)}$. However, the differences in the change among these six major serum carotenoid concentrations against oxidative stress induced by cigarette smoking and alcohol drinking have not been thoroughly studied while controlling for dietary carotenoid concentrations. 
The present study aimed to investigate the interaction of cigarette smoking and alcohol drinking with the serum concentrations of the following main six carotenoid concentrations, i.e. lutein, lycopene, $\alpha$-carotene, $\beta$-carotene, $\beta$-cryptoxanthin and zeaxanthin. The synergistic interaction of cigarette smoking and alcohol drinking with serum carotenoid concentrations was evaluated cross-sectionally.

\section{Subjects and methods}

\section{Subjects}

Data used in the present study were derived from health examinations of residents of the town of Mikkabi, Shizuoka Prefecture, Japan, aged from 30 to 70 years, in 2003 and 2005. Mikkabi is located in western Shizuoka, and about $40 \%$ of its residents work in agriculture. In 2003, a total of 1979 males and females were subjects for the health examination. As a result, 1448 participants (73.2\% of the total) received a health examination. Informed consent was obtained from the 886 subjects ( 302 male and 584 female) recruited for the present study. The response rate was $61.2 \%$. In 2005, a total of 1891 males and females were subjects for the health examinations. As a result, 1369 participants $(72.4 \%$ of total subjects) underwent such an examination. Participants who had received the health examination in 2005 were further recruited for the present study, and informed consent was newly obtained from 187 subjects (fifty-five male and 132 female). As a result, a total of 1073 subjects were included in this survey.

In the present study, the following subjects were excluded from the data analysis: (1) those for whom the self-administered questionnaire data were incomplete; and (2) those for whom blood samples for serum carotenoid analysis were not collected. As a result, a total of 354 male and 715 female subjects were included for further data analysis.

\section{Measurements}

Blood samples were obtained in the morning after overnight fasting. Serum was separated from blood cells by centrifugation and stored at $-80^{\circ} \mathrm{C}$ until analysis of the serum carotenoid concentrations. The concentrations of six serum carotenoids (lutein, lycopene, $\alpha$-carotene, $\beta$-carotene, $\beta$-cryptoxanthin and zeaxanthin) were analysed by reverse-phase HPLC using $\beta$-apo- $8^{\prime}$-carotenal as an internal standard at the laboratory of Public Health and Environmental Chemistry, Kyoto Biseibutsu Kenkyusho (Kyoto, Japan), as described previously ${ }^{(33)}$. The serum total cholesterol was measured using an autoanalyser using a commercial kit (Determiner TC-II C for serum total cholesterol; Kyowa-Medics, Inc., Tokyo, Japan) at the laboratory of the Seirei Preventive Health Care Centre (Shizuoka, Japan). Height and body weight were measured by trained public health nurses. BMI was calculated as the body weight (kg) divided by the height $\left(\mathrm{m}^{2}\right)$.

\section{Lifestyle assessment and dietary data analysis}

A self-administered questionnaire was used to collect lifestyle information, including tobacco use (current smoker, exsmoker, or non-smoker), exercise (weekly participation), regular alcohol intake (one or more times per week) and dietary habits. The assessment of diet was a modification of the validated self-administered 121-item simple FFQ developed especially for the Japanese by Wakai and colleagues ${ }^{(34,35)}$. Information about alcohol consumption from Japanese sake, beer, shochu, wine and whisky, and the daily intake of eighteen nutrients from foods were estimated from monthly food intake frequencies with either standard portion size (for most types of food) or subject-specified usual portion size (for rice, bread, and alcoholic and non-alcoholic beverages) using the FFQ analysis software package for windows (Food Frequency Questionnaire System; System Supply Co., Ltd, Kanagawa, Japan). This FFQ analysis software computes an individual's food and nutrient intake from FFQ data on the basis of the Standard Tables of Food Composition in Japan $^{(36)}$. The dietary carotenoid intakes of each individual were computed to obtain the amount of six carotenoids (lycopene, $\alpha$-carotene, $\beta$-carotene, lutein, $\beta$-cryptoxanthin and zeaxanthin) using a published database of the carotenoid composition of fruit and vegetables ${ }^{(37,38)}$. In our survey, we calculated an individual's carotenoid intake from important sources of carotenoids. In this data analysis, the dietary carotenoid intakes were calculated from the FFQ data of each individual's food items, not dishes. The dietary intakes of six carotenoids and total energy intake of all subjects were used in the present report.

\section{Statistical analysis}

The dietary intakes and serum concentrations of carotenoids were skewed toward higher concentrations. These values were $\log$ (natural)-transformed to improve the normality of their distribution. The $t$ test was used to compare the means of continuous variables in two groups. The $\chi^{2}$ test was used to compare the rates of categorical variables in two groups. All variables were presented as an original scale. The data are expressed as the mean values and standard deviations, geometric mean values with $95 \% \mathrm{CI}$, range, or percentages. In order to examine the relationship of independent variables with each serum carotenoid concentration, multiple regression analysis was performed with sex, age, BMI, smoking status, ethanol intake, serum total cholesterol, total energy intake excluding ethanol, and dietary intakes of respective carotenoids included always in the models as independent variables. The subjects were divided into three groups stratified by alcohol intake levels defined as non-drinker $(<1 \mathrm{~g}$ ethanol per $\mathrm{d})$, light drinker ( $\geq 1$ to $<25 \mathrm{~g}$ ethanol per $\mathrm{d}$ ) and moderate and heavy drinkers ( $\geq 25 \mathrm{~g}$ ethanol per $\mathrm{d}$ ) because one glass of Japanese sake $(180 \mathrm{ml})$ or one bottle of beer $(633 \mathrm{ml})$, which are major alcohol beverages and widely consumed in Japan, commonly contains about $25 \mathrm{~g}$ alcohol. In our study population, the number of ex-smokers was ninety-seven $(9.1 \%$ among the study population) from the self-administered questionnaire. However, in our survey, we did not collect data on time since last smoking. Therefore, ex-smokers were included in the non-smokers group. All subjects were categorised into six groups according to daily alcohol intake (non-drinkers, $<1 \mathrm{~g} / \mathrm{d}$; light drinkers, $\geq 1$ to $<25 \mathrm{~g} / \mathrm{d}$; moderate-to-heavy drinkers, $\geq 25 \mathrm{~g} / \mathrm{d}$ ) and smoking status (current smokers and non-smokers, including ex-smokers). The multivariate adjusted mean of the dietary intakes and serum concentrations 
of six carotenoids were calculated after adjusting for confounding factors. Differences in the multivariate adjusted mean of the dietary intakes and serum concentrations of six carotenoids among each group were tested by Bonferroni multiple comparison.

In our study population, the subjects who take carotenoid supplements were only $0.2 \%$ among the study population. Therefore, we did not take account of carotenoid intake from supplements in the data analysis. The detection limit for the serum lycopene concentration for the method used in the study was $0.04 \mu \mathrm{g} / \mathrm{ml}(0.075 \mu \mathrm{mol} / \mathrm{l})$, and values below the limit of detection of the assay were marked as $0.03 \mu \mathrm{g} / \mathrm{ml}(0.056 \mu \mathrm{mol} / \mathrm{l})$ in the analysis. Each of the detection limits of other carotenoids was $0.02 \mu \mathrm{g} / \mathrm{ml}$. In our survey, except for lycopene, there was no subject whose serum concentration of carotenoid was under the limit of detection. All statistical analyses were performed using the statistical software package SPSS for Windows (version 12.0J; SPSS Inc., Chicago, IL, USA) on a personal computer.

\section{Ethical approval}

The present study was conducted according to the guidelines laid down in the Declaration of Helsinki and all procedures involving human subjects were approved by the ethics committee of the National Institute of Fruit Tree Science and the Hamamatsu University School of Medicine. Written informed consent was obtained from all subjects.

\section{Results}

Table 1 shows the characteristics of the study subjects stratified by sex. The dietary carotenoid intakes, excluding $\beta$-cryptoxanthin, were significantly higher in female than in male subjects. The serum carotenoid concentrations, excluding zeaxanthin, were significantly higher in female than in male subjects. The rates of current smokers and regular alcohol drinkers were higher in male than in female subjects.

Table 1. Characteristics of the study subjects

(Geometric means and 95\% confidence intervals, mean values and standard deviations or percentages)

\begin{tabular}{|c|c|c|c|c|}
\hline & \multicolumn{2}{|c|}{ Male (n 354) } & \multicolumn{2}{|c|}{ Female ( $n 715)$} \\
\hline & Geometric mean & $95 \% \mathrm{Cl}$ & Geometric mean & $95 \% \mathrm{Cl}$ \\
\hline \multicolumn{5}{|l|}{ Age (years) } \\
\hline Mean & \multicolumn{2}{|l|}{$56 \cdot 3$} & \multicolumn{2}{|l|}{$54 \cdot 6^{\star \star}$} \\
\hline $\mathrm{SD}$ & \multicolumn{2}{|l|}{9.7} & \multicolumn{2}{|l|}{$10 \cdot 1$} \\
\hline \multicolumn{5}{|l|}{ BMI $\left(\mathrm{kg} / \mathrm{m}^{2}\right)$} \\
\hline Mean & \multicolumn{2}{|l|}{23.5} & \multicolumn{2}{|l|}{$22 \cdot 6^{\star \star \star}$} \\
\hline SD & \multicolumn{2}{|l|}{2.9} & \multicolumn{2}{|l|}{$3 \cdot 1$} \\
\hline \multicolumn{5}{|l|}{ Total cholesterol $(\mathrm{mmol} / \mathrm{l})$} \\
\hline Mean & \multirow{2}{*}{\multicolumn{2}{|c|}{$\begin{array}{l}5 \cdot 31 \\
0.86\end{array}$}} & \multicolumn{2}{|l|}{$5 \cdot 61^{* \star *}$} \\
\hline SD & & & \multicolumn{2}{|l|}{0.90} \\
\hline \multicolumn{3}{|l|}{ Total energy intake $(\mathrm{kJ} / \mathrm{d})$} & & \\
\hline Including ethanol & \multirow{2}{*}{\multicolumn{2}{|c|}{9128.9}} & & \\
\hline Mean & & & \multicolumn{2}{|l|}{$8370 \cdot 0^{\star \star \star}$} \\
\hline SD & \multicolumn{2}{|l|}{$2277 \cdot 4$} & \multicolumn{2}{|l|}{$2362 \cdot 8$} \\
\hline \multicolumn{5}{|l|}{ Excluding ethanol } \\
\hline Mean & \multicolumn{2}{|l|}{$8380 \cdot 4$} & \multicolumn{2}{|l|}{$8319 \cdot 1$} \\
\hline SD & \multicolumn{2}{|l|}{$2152 \cdot 0$} & \multicolumn{2}{|l|}{$2364 \cdot 8$} \\
\hline \multicolumn{5}{|l|}{ Ethanol intake (g/d) } \\
\hline Mean & $26 \cdot 9$ & & $1 \cdot 6^{\star \star \star}$ & \\
\hline SD & $31 \cdot 2$ & & 4.9 & \\
\hline Dietary carotenoid intakes ( $\mathrm{n}$ & & & & \\
\hline Lycopene & 0.05 & $0.04,0.06$ & $0.07^{\star \star}$ & $0.06,0.08$ \\
\hline$\alpha$-Carotene & 0.11 & $0.10,0.12$ & $0.24^{* * *}$ & $0.23,0.26$ \\
\hline$\beta$-Carotene & 1.06 & $0.99,1.13$ & $1 \cdot 70^{\star \star \star}$ & $1.62,1.77$ \\
\hline Lutein & 1.38 & $1.30,1.47$ & $1.92^{\star \star *}$ & $1 \cdot 84,2 \cdot 00$ \\
\hline$\beta$-Cryptoxanthin & 0.30 & $0.25,0.37$ & 0.31 & $0.27,0.37$ \\
\hline Zeaxanthin & 0.59 & $0.55,0.65$ & $0 \cdot 70^{\star \star *}$ & $0.67,0.75$ \\
\hline Serum carotenoid concentra & $(\mu \mathrm{mol} / \mathrm{l})$ & & & \\
\hline Lycopene & 0.21 & $0.19,0.23$ & $0.31^{\star \star \star}$ & $0.29,0.33$ \\
\hline$\alpha$-Carotene & $0 \cdot 10$ & $0.10,0.11$ & $0.15^{\star \star \star}$ & $0.14,0.16$ \\
\hline$\beta$-Carotene & 0.40 & $0.38,0.43$ & $0.74^{\star \star \star}$ & $0.71,0.77$ \\
\hline Lutein & 0.52 & $0.50,0.55$ & $0.57^{\star \star}$ & $0.55,0.58$ \\
\hline$\beta$-Cryptoxanthin & 1.03 & $0.93,1.14$ & $1.46^{* * *}$ & $1.37,1.55$ \\
\hline Zeaxanthin & 0.22 & $0.22,0.23$ & 0.23 & $0.22,0.23$ \\
\hline Habitual exercise (\%)‡ & $22 \cdot 1$ & & $23 \cdot 2$ & \\
\hline Current smoker (\%) & $33 \cdot 1$ & & $1.4+\dagger \dagger$ & \\
\hline Regular alcohol intake (\%)‡ & 60.5 & & $9 \cdot 4 \dagger \dagger \dagger$ & \\
\hline
\end{tabular}

Mean value was significantly different from that for the male subjects: ${ }^{* \star} P<0.01,{ }^{\star \star *} P<0.001$ (Student's $t$ test). $\dagger \dagger \dagger$ Percentage was significantly different from that for the male subjects $\left(P<0.001 ; \chi^{2}\right.$ test). $\ddagger$ One or more times/week. 
Next, in order to examine the relationship of independent variables with each serum carotenoid concentration, multiple regression analysis was performed; sex, age, BMI, smoking status, ethanol intake, serum total cholesterol, total energy intake excluding ethanol, and dietary intakes of carotenoids were always included in the models as independent variables (Table 2). All six serum carotenoid concentrations were significantly associated with the dietary intakes of carotenoids. The strongest correlation between dietary intake and serum concentration was observed in $\beta$-cryptoxanthin. The serum total cholesterol was a significant positive predictor of the serum carotenoid concentration. The BMI was a significant negative predictor of serum carotenoid concentrations excluding $\beta$-cryptoxanthin. Smoking status was a significant negative predictor of the serum carotenoid concentration except for zeaxanthin. Ethanol intake was a significant negative predictor of the concentrations of serum lycopene, $\alpha$-carotene, $\beta$-carotene and $\beta$-cryptoxanthin. Neither smoking status nor ethanol intake was associated with the serum zeaxanthin concentration.

Table 3 shows the results of the dietary carotenoid intakes in six groups stratified by daily alcohol intake and smoking status. The dietary intakes of $\alpha$ - and $\beta$-carotene in current smokers and/or in regular alcohol drinkers $(>1 \mathrm{~g} / \mathrm{d})$ were significantly lower than those in non-smokers among nondrinkers. In addition, the dietary intake of lutein was significantly lower in moderate-to-heavy drinkers (more than $25 \mathrm{~g} / \mathrm{d}$ ) and in light drinkers (1-25g/d) among current smokers than it was in non-smokers among non-drinkers. The dietary intake of $\beta$-cryptoxanthin was significantly lower in light drinkers $(1-25 \mathrm{~g} / \mathrm{d})$ among current smokers than it was in non-smokers among non-drinkers. However, after adjusting for age and sex, these significantly lower dietary intakes of carotenoids were non-significant.

The unadjusted and adjusted means of the serum carotenoid concentration among the six groups are shown in Table 4. After adjusting for age and sex, the serum lutein and zeaxanthin concentrations were not different among the six groups stratified by daily alcohol intake and smoking status. In contrast, the serum lycopene concentration in moderate-to-heavy drinkers was significantly lower than that in non-drinkers. Although this lower serum lycopene concentration in moderate-to-heavy drinkers was observed in both non-smokers and current smokers, no significant difference was observed between non-smokers and current smokers. On the other hand, the concentrations of serum $\alpha$-carotene, $\beta$-carotene and $\beta$-cryptoxanthin were significantly lower than those with increased alcohol intake. Furthermore, the serum $\alpha$-carotene, $\beta$-carotene and $\beta$-cryptoxanthin of current smokers among regular alcohol drinkers (more than $1 \mathrm{~g} / \mathrm{d}$ ) were significantly lower than those of non-smokers who have the same alcohol intake. The lower serum carotenoid concentrations among regular alcohol drinkers were more evident in current smokers than in non-smokers. After further adjusting for BMI, total cholesterol, total energy intake excluding alcohol, and dietary intake of respective carotenoids, no obvious change in these associations of cigarette smoking and alcohol drinking with the serum carotenoid concentration was observed.

Furthermore, after multivariate adjusting, no obvious change of the present results about the differences in the

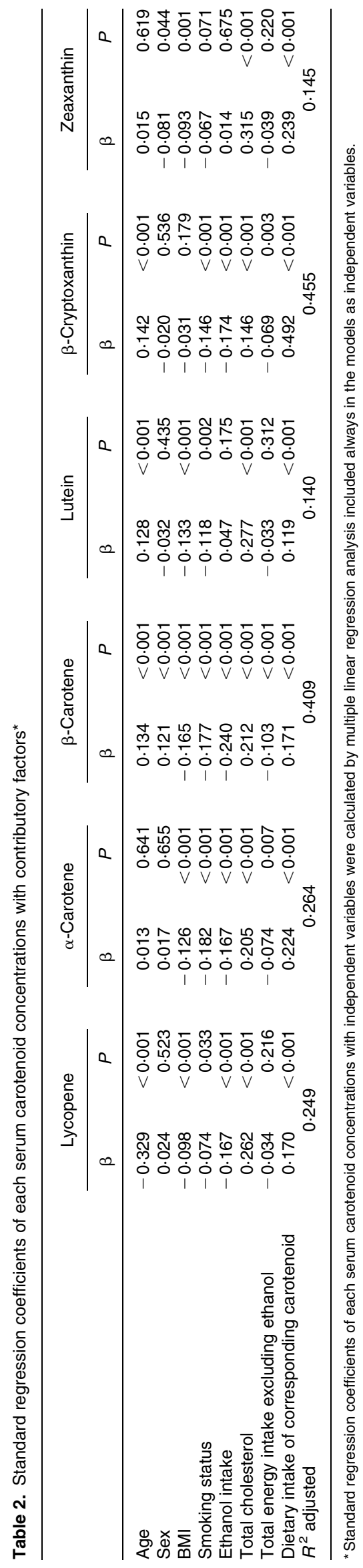


Table 3. Unadjusted and adjusted dietary carotenoid intakes stratified by smoking status and daily alcohol intake (Geometric means and $95 \%$ confidence intervals, mean values and standard deviations or percentages)

\begin{tabular}{|c|c|c|c|c|c|c|c|c|c|c|c|c|}
\hline & \multicolumn{12}{|c|}{ Daily alcohol intake } \\
\hline & \multicolumn{4}{|c|}{$<1 \mathrm{~g} / \mathrm{d}$} & \multicolumn{4}{|c|}{$\geq 1$ to $<25 \mathrm{~g} / \mathrm{d}$} & \multicolumn{4}{|c|}{$\geq 25 \mathrm{~g} / \mathrm{d}$} \\
\hline & \multicolumn{2}{|c|}{ Non-smokers ( $n$ 579) } & \multicolumn{2}{|c|}{ Current-smokers ( $n$ 29) } & \multicolumn{2}{|c|}{ Non-smokers ( $n$ 263) } & \multicolumn{2}{|c|}{ Current-smokers (n 35) } & \multicolumn{2}{|c|}{ Non-smokers $(n 100)$} & \multicolumn{2}{|c|}{ Current-smokers ( $n$ 63) } \\
\hline & $\begin{array}{c}\text { Geometri } \\
\text { mean }\end{array}$ & $95 \% \mathrm{Cl}$ & $\begin{array}{c}\text { Geometric } \\
\text { mean }\end{array}$ & $95 \% \mathrm{Cl}$ & $\begin{array}{c}\text { Geometric } \\
\text { mean }\end{array}$ & $95 \% \mathrm{Cl}$ & $\begin{array}{c}\text { Geometric } \\
\text { mean }\end{array}$ & $95 \% \mathrm{Cl}$ & $\begin{array}{c}\text { Geometric } \\
\text { mean }\end{array}$ & $95 \% \mathrm{Cl}$ & $\begin{array}{c}\text { Geometri } \\
\text { mean }\end{array}$ & $95 \% \mathrm{Cl}$ \\
\hline \multicolumn{13}{|l|}{ Age (years) } \\
\hline Mean & \multicolumn{2}{|c|}{$55 \cdot 8$} & \multicolumn{2}{|c|}{53.4} & \multicolumn{2}{|c|}{$53 \cdot 8$} & \multicolumn{2}{|c|}{$49 \cdot 3^{\star \star}$} & \multicolumn{2}{|c|}{$57 \cdot 1$} & \multicolumn{2}{|c|}{$55 \cdot 6$} \\
\hline SD & \multirow{2}{*}{\multicolumn{2}{|c|}{$\begin{array}{l}9.9 \\
7.8\end{array}$}} & \multirow{2}{*}{\multicolumn{2}{|c|}{$\begin{array}{l}10 \cdot 0 \\
82.8\end{array}$}} & \multirow{2}{*}{\multicolumn{2}{|c|}{$10 \cdot 2$}} & \multicolumn{2}{|c|}{$9 \cdot 3$} & \multicolumn{2}{|c|}{$9 \cdot 6$} & & \\
\hline Male (\%) & & & & & & & & & & & & \\
\hline $\mathrm{BMI}\left(\mathrm{kg} / \mathrm{m}^{2}\right)$ & & & & & & & & & & & & \\
\hline Mean & & & & & & & & & & & & \\
\hline SD & & & & & & & & & & & & \\
\hline Serum total ch & sterol $(\mathrm{mm}$ & & & & & & & & & & & \\
\hline Mean & & & & & & & & & & & & \\
\hline SD & & & & & & & & & & & & \\
\hline Total energy in & $\mathrm{ke}(\mathrm{kJ} / \mathrm{d})$ & & & & & & & & & & & \\
\hline Mean & & & & & & & & & & & & \\
\hline $\mathrm{SD}$ & & & & & & & & & & & & \\
\hline Excluding et & & & & & & & & & & & & \\
\hline Mean & & & & & & & & & & & & \\
\hline SD & & & & & & & & & & & & \\
\hline Ethanol intake & & & & & & & & & & & & \\
\hline Mean & & & & & & & & & & & & \\
\hline SD & & & & & & & & & & & & \\
\hline Dietary caroten & $d$ intakes ( $n$ & & & & & & & & & & & \\
\hline Lycopene & & & & & & & & & & & & \\
\hline Crude & 0.07 & $0.06,0.08$ & 0.05 & $0.02,0.12$ & 0.07 & $0.05,0.08$ & 0.04 & $0.02,0.08$ & 0.06 & $0.04,0.08$ & 0.04 & $0.03,0.07$ \\
\hline $\begin{array}{c}\text { Adjusted } † \\
\alpha \text {-Carotene }\end{array}$ & 0.06 & $0.05,0.07$ & 0.06 & $0.03,0.13$ & 0.07 & $0.05,0.09$ & 0.05 & $0.02,0.09$ & 0.07 & $0.04,0.10$ & 0.05 & $0.03,0.09$ \\
\hline Crude & 0.23 & $0.21,0.24$ & $0.14^{\star}$ & $0 \cdot 10,0.19$ & $0 \cdot 18^{\star \star}$ & $0.16,0.20$ & $0.14^{*}$ & $0.10,0.20$ & $0 \cdot 12^{\star \star \star}$ & $0.10,0.14$ & $0.09^{\star \star \star}$ & $0.07,0.12$ \\
\hline Adjusted $\dagger$ & 0.19 & $0.18,0.21$ & 0.20 & $0.15,0.27$ & $0 \cdot 19$ & $0.17,0.21$ & 0.21 & $0.16,0.28$ & 0.18 & $0.15,0.22$ & 0.15 & $0 \cdot 12,0.18$ \\
\hline$\beta$-Carotene & & & & & & & & & & & & \\
\hline Crude & 1.68 & $1 \cdot 60,1.76$ & $1 \cdot 17^{\star \star}$ & $0.89,1.53$ & $1 \cdot 37^{\star \star \star}$ & $1 \cdot 26,1.48$ & $1.04^{\star \star \star}$ & $0.80,1.36$ & $1 \cdot 10^{\star \star \star}$ & $0.98,1.23$ & $0.99^{\star \star \star}$ & $0.83,1.17$ \\
\hline Adjusted† & 1.49 & $1.40,1.58$ & 1.49 & $1 \cdot 18,1.87$ & 1.42 & $1 \cdot 31,1.52$ & 1.42 & $1 \cdot 14,1 \cdot 75$ & 1.41 & $1 \cdot 23,1.61$ & 1.32 & $1 \cdot 11,1.56$ \\
\hline Lutein & & & & & & & & & & & & \\
\hline Crude & 1.89 & $1.80,1.99$ & 1.51 & $1.19,1.92$ & 1.68 & $1.56,1 \cdot 80$ & $1.34^{*}$ & $1.06,1 \cdot 70$ & $1.37^{\star \star \star}$ & $1.23,1.54$ & $1.40^{\star *}$ & $1.20,1.65$ \\
\hline Adjusted† & 1.74 & $1.64,1.83$ & 1.79 & $1.43,2.23$ & 1.72 & $1.60,1.85$ & 1.66 & $1.35,2.04$ & 1.65 & $1.44,1.88$ & 1.73 & $1.47,2.03$ \\
\hline$\beta$-Cryptoxan & & & & & & & & & & & & \\
\hline Crude & 0.39 & $0.33,0.46$ & $0 \cdot 16$ & $0.07,0.41$ & 0.25 & $0.19,0.33$ & $0 \cdot 10^{* *}$ & $0.04,0.25$ & 0.30 & $0.20,0.45$ & 0.25 & $0.16,0.41$ \\
\hline Adjusted $\dagger$ & 0.38 & $0.32,0.45$ & $0 \cdot 18$ & $0.09,0.36$ & 0.28 & $0.22,0.36$ & $0 \cdot 16$ & $0.08,0.30$ & 0.23 & $0.15,0.35$ & 0.22 & $0.13,0.37$ \\
\hline Zeaxanthin & & & & & & & & & & & & \\
\hline Crude & 0.69 & $0.64,0.74$ & 0.55 & $0.40,0.75$ & 0.68 & $0.62,0.75$ & 0.62 & $0.48,0.80$ & 0.57 & $0.49,0.66$ & 0.65 & $0.52,0.80$ \\
\hline Adjusted $\dagger$ & 0.66 & $0.61,0.71$ & 0.59 & $0.44,0.80$ & 0.68 & $0.62,0.75$ & 0.67 & $0.50,0.88$ & 0.63 & $0.53,0.75$ & 0.72 & $0.58,0.89$ \\
\hline
\end{tabular}

Mean value was significantly different from that of non-smoking non-drinkers: ${ }^{*} P<0.05,{ }^{* *} P<0.01,{ }^{* *} P<0.001$ (Bonferroni multiple comparison test).

$\dagger$ Age and sex were adjusted. 
Table 4. Unadjusted and adjusted serum carotenoid concentrations stratified by smoking status and daily alcohol intake (Geometric means and $95 \%$ confidence intervals)

\begin{tabular}{|c|c|c|c|c|c|c|c|c|c|c|c|c|}
\hline \multirow[b]{4}{*}{$\begin{array}{l}\text { Serum carotenoids } \\
(\mu \mathrm{mol} / \mathrm{l})\end{array}$} & \multicolumn{12}{|c|}{ Daily alcohol intake } \\
\hline & \multicolumn{4}{|c|}{$<1 \mathrm{~g} / \mathrm{d}$} & \multicolumn{4}{|c|}{$\geq 1$ to $<25 \mathrm{~g} / \mathrm{d}$} & \multicolumn{4}{|c|}{$\geq 25 \mathrm{~g} / \mathrm{d}$} \\
\hline & \multicolumn{2}{|c|}{ Non-smokers } & \multicolumn{2}{|c|}{ Current-smokers } & \multicolumn{2}{|c|}{ Non-smokers } & \multicolumn{2}{|c|}{ Current-smokers } & \multicolumn{2}{|c|}{ Non-smokers } & \multicolumn{2}{|c|}{ Current-smokers } \\
\hline & $\begin{array}{c}\text { Geometric } \\
\text { mean }\end{array}$ & $95 \% \mathrm{Cl}$ & $\begin{array}{l}\text { Geometric } \\
\text { mean }\end{array}$ & $95 \% \mathrm{Cl}$ & $\begin{array}{l}\text { Geometric } \\
\text { mean }\end{array}$ & $95 \% \mathrm{Cl}$ & $\begin{array}{l}\text { Geometric } \\
\text { mean }\end{array}$ & $95 \% \mathrm{Cl}$ & $\begin{array}{c}\text { Geometric } \\
\text { mean }\end{array}$ & $95 \% \mathrm{Cl}$ & $\begin{array}{l}\text { Geometric } \\
\text { mean }\end{array}$ & $95 \% \mathrm{Cl}$ \\
\hline \multicolumn{13}{|l|}{ Lycopene } \\
\hline Crude & 0.30 & $0.29,0.32$ & 0.24 & $0 \cdot 19,0.31$ & 0.29 & $0.27,0.32$ & 0.21 & $0.17,0.28$ & $0 \cdot 19^{\star \star \star}$ & $0.16,0.22$ & $0 \cdot 17^{\star \star *}$ & $0.14,0.20$ \\
\hline Adjusted§ & 0.29 & $0.28,0.31$ & 0.26 & $0.20,0.33$ & 0.28 & $0.26,0.31$ & 0.21 & $0.17,0.26$ & $0 \cdot 21^{\star \star}$ & $0.18,0.25$ & $0 \cdot 19^{\star \star *}$ & $0.15,0.22$ \\
\hline Adjusted\| & 0.30 & $0.28,0.32$ & 0.24 & $0.19,0.31$ & 0.28 & $0.26,0.30$ & $0.20^{*}$ & $0.16,0.25$ & $0 \cdot 21^{\star \star \star}$ & $0.18,0.24$ & $0.19^{* \star *}$ & $0.16,0.22$ \\
\hline \multicolumn{13}{|l|}{$\alpha$-Carotene } \\
\hline Crude & 0.14 & $0 \cdot 14,0 \cdot 15$ & $0 \cdot 10^{\star *}$ & $0.09,0.12$ & 0.13 & $0.13,0.14$ & $0.09^{\star \star \star}+\dagger \dagger$ & $0.08,0.11$ & $0 \cdot 09^{\star \star \star}$ & $0.09,0.10$ & 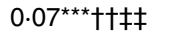 & $0.06,0.08$ \\
\hline Adjusted§ & $0 \cdot 14$ & $0.13,0.14$ & $0 \cdot 11$ & $0 \cdot 10,0.14$ & $0 \cdot 14$ & $0.13,0.14$ & $0 \cdot 10^{*} \dagger$ & $0.09,0.12$ & $0 \cdot 11^{\star \star}$ & $0.10,0.12$ & $0 \cdot 08^{\star \star \star} \dagger † \ddagger$ & $0.07,0.09$ \\
\hline Adjusted\|| & $0 \cdot 14$ & $0.13,0.14$ & $0 \cdot 11$ & $0.09,0.13$ & $0 \cdot 13$ & $0.13,0.14$ & $0 \cdot 10^{* *} \dagger \dagger$ & $0.09,0.12$ & $0 \cdot 11^{\star \star \star}$ & $0.10,0.12$ & $0.08^{\star \star \star} \dagger \dagger$ & $0.07,0.09$ \\
\hline \multicolumn{13}{|l|}{$\beta$-Carotene } \\
\hline Crude & 0.74 & $0.71,0.77$ & $0.50^{\star \star}$ & $0.41,0.60$ & $0 \cdot 63^{\star \star}$ & $0.59,0.67$ & $0.35^{\star \star \star}+\dagger \dagger$ & $0.28,0.45$ & $0.38^{\star \star *}$ & $0.34,0.42$ & 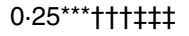 & $0.22,0.29$ \\
\hline Adjusted§ & 0.66 & $0.63,0.70$ & 0.61 & $0.51,0.74$ & 0.65 & $0.61,0.70$ & $0.47^{\star \star} \dagger$ & $0.40,0.57$ & $0.46^{\star \star \star}$ & $0.41,0.52$ & 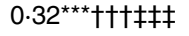 & $0.28,0.37$ \\
\hline Adjusted\| & 0.67 & $0 \cdot 64,0.70$ & 0.58 & $0.49,0.70$ & 0.65 & $0.61,0.69$ & $0.46^{* * \star}+\dagger$ & $0.39,0.54$ & $0.46^{\star \star \star}$ & $0.41,0.51$ & 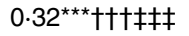 & $0.28,0.36$ \\
\hline \multicolumn{13}{|l|}{ Lutein } \\
\hline Crude & 0.60 & $0.58,0.62$ & 0.52 & $0.45,0.61$ & 0.59 & $0.57,0.62$ & $0.47^{\star \star}+\dagger$ & $0.40,0.54$ & 0.59 & $0.55,0.64$ & 0.55 & $0.50,0.61$ \\
\hline Adjusted§ & 0.58 & $0.56,0.60$ & 0.56 & $0.49,0.64$ & 0.60 & $0.58,0.63$ & 0.52 & $0.45,0.59$ & 0.62 & $0.57,0.67$ & 0.59 & $0.53,0.66$ \\
\hline Adjusted\| & 0.58 & $0.56,0.60$ & 0.54 & $0.47,0.62$ & 0.60 & $0.57,0.63$ & 0.51 & $0.45,0.57$ & 0.62 & $0.57,0.67$ & 0.59 & $0.53,0.65$ \\
\hline \multicolumn{13}{|l|}{$\beta$-Cryptoxanthin } \\
\hline Crude & 1.55 & $1.45,1.65$ & 1.01 & $0.71,1.44$ & 1.34 & $1 \cdot 21,1 \cdot 48$ & $0 \cdot 62^{\star \star \star}+\dagger \dagger$ & $0.44,0.86$ & $0.98^{\star \star \star}$ & $0.82,1 \cdot 17$ & $0.61^{* \star \star} \dagger \dagger$ & $0.49,0.76$ \\
\hline Adjusted§ & 1.49 & $1.38,1.60$ & $1 \cdot 11$ & $0.83,1.48$ & 1.41 & $1.28,1.55$ & $0.78^{\star \star \star} \dagger \dagger \dagger$ & $0.60,1.02$ & $0.95^{\star \star \star}$ & $0.80,1 \cdot 13$ & $0.62^{\star \star \star} \dagger \dagger \ddagger$ & $0.50,0.77$ \\
\hline Adjusted\| & 1.44 & $1.35,1.53$ & 1.20 & $0.94,1.54$ & 1.43 & $1.32,1.55$ & $0.87^{\star \star}+\dagger \dagger$ & $0.70,1 \cdot 10$ & $1.00^{\star \star \star}$ & $0.87,1 \cdot 16$ & 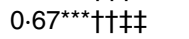 & $0.56,0.80$ \\
\hline \multicolumn{13}{|l|}{ Zeaxanthin } \\
\hline Crude & 0.24 & $0.24,0.25$ & 0.23 & $0.21,0.27$ & 0.25 & $0.24,0.26$ & 0.22 & $0.20,0.24$ & 0.24 & $0.23,0.26$ & 0.24 & $0.22,0.26$ \\
\hline Adjusted§ & 0.24 & $0.23,0.25$ & 0.24 & $0.21,0.27$ & 0.25 & $0.24,0.26$ & 0.23 & $0.20,0.25$ & 0.24 & $0.23,0.26$ & 0.24 & $0.22,0.26$ \\
\hline Adjusted\| & 0.24 & $0.23,0.25$ & 0.24 & $0.21,0.26$ & 0.25 & $0.24,0.25$ & 0.22 & $0.20,0.25$ & 0.24 & $0.23,0.26$ & 0.24 & $0.22,0.26$ \\
\hline
\end{tabular}

Mean value was significantly different from that of non-smoking non-drinkers: ${ }^{\star} P<0.05,{ }^{* \star} P<0.01,{ }^{* \star \star} P<0.001$ (Bonferroni multiple comparison test).

Mean value was significantly different from that of non-smokers who have the same alcohol intake: $\dagger P<0.05, \dagger \dagger P<0.01, t+\dagger P<0.001$ (Bonferroni multiple comparison test).

Mean value was significantly different from that of non-drinking current smokers: $\ddagger P<0.05$, $\neq \ddagger P<0.01, \ddagger \neq \ddagger P<0.001$ (Bonferroni multiple comparison test).

$\S$ Age and sex were adjusted.

\| Age, sex, BMI, total cholesterol, total energy intake excluding alcohol, and dietary intake of corresponding carotenoid were adjusted. 
changes among the serum concentrations of six carotenoids stratified by cigarette smoking and alcohol drinking habits were observed, even though female subjects were excluded (data not shown).

\section{Discussion}

The original FFQ we used in the present survey is not able to estimate the amount of carotenoid intake from foods because all six carotenoid contents in each food are not assessed in standard tables of food composition in Japan. However, recently, a database of the carotenoid composition of fruit and vegetables was published ${ }^{(37,38)}$. In the present study, we adapted FFQ analysis software package to calculate an individual's carotenoid intake from important sources of carotenoids, and significant relationships between dietary intake of each carotenoid and its serum levels were observed. This is the first experiment to estimate six dietary carotenoids in Japan.

The present investigation is the first-reported cross-sectional study to examine the synergistic interaction of cigarette smoking and alcohol drinking with the six major serum carotenoids while controlling for dietary carotenoid intakes. The results indicated that daily alcohol intake may reduce the serum concentrations of lycopene, $\alpha$-carotene, $\beta$-carotene and $\beta$-cryptoxanthin in a dose-dependent manner. Furthermore, these alcohol-related lower serum carotenoid concentrations, except for lycopene, were more evident in current smokers than in non-smokers. The serum lycopene concentration seems to be influenced by alcohol drinking and not by smoking. In addition, neither smoking nor alcohol drinking affected the serum lutein and zeaxanthin concentrations.

Although many epidemiological studies have indicated that the serum carotenoid concentrations were low among current smokers and/or regular alcohol drinkers ${ }^{(19-31)}$, most of these previous studies evaluated the effect of these lifestyle factors on the serum carotenoid concentrations separately. It is widely known that the dietary intake of carotenoids is lower in smokers than in non-smokers, in alcohol drinkers than in non-drinkers, and in males than in females ${ }^{(21)}$. In addition, the consumption of alcohol among smokers is higher than that among non-smokers. Therefore, the possibility that the correlation between cigarette smoking and alcohol drinking with the serum carotenoid concentrations observed in these previous reports would be an artifact of the strong correlation of cigarette smoking and alcohol drinking could not be ruled out. In our data analysis, the subjects were stratified into six groups according to their smoking status and the amount of daily alcohol intake. Thus, we believe that the influence of the association among cigarette smoking and alcohol drinking can be completely eliminated in the analysis of these six subgroups.

Previously, seven cross-sectional studies have reported the possibility that cigarette smoking and alcohol drinking might synergistically enhance the depletion of serum carotenoid concentrations $^{(19-23,25,26)}$. However, these studies did not take into account the dietary intakes of carotenoids; therefore, it is not clear whether the low serum carotenoid concentration was due to the low dietary intake of carotenoids or whether cigarette smoking and alcohol drinking would cause deterioration in the serum carotenoid concentrations.
To our knowledge, there have been no reports about the synergistic interaction of cigarette smoking and alcohol drinking with the serum carotenoid concentrations after taking into account the dietary carotenoid intakes. Furthermore, there is limited information about the differences in the changes among the serum concentrations of the six major carotenoids against oxidative stress induced by cigarette smoking and alcohol drinking. In our data analysis, the dietary intakes of $\alpha$-carotene, $\beta$-carotene and lutein in current smokers and/or regular alcohol drinkers were significantly lower than those in non-smokers among non-drinkers. However, these significantly lower dietary intakes became insignificant after adjusting for age and sex. Furthermore, a significant synergistic interaction of cigarette smoking and alcohol drinking with the serum carotenoid concentrations was observed after further adjusting for the dietary intakes of respective carotenoids. Therefore, it seems that the significant differences of the multivariate adjusted means of the serum carotenoid concentrations among the six groups stratified by lifestyle factors might be caused by the depletion of serum carotenoids and not by differences in dietary carotenoid intakes.

Recently, some animal experiments have been reported concerning the synergistic effect of cigarette and alcohol on the antioxidant defence system in several tissues ${ }^{(39-41)}$. These animal experiments show the possibility that combination of alcohol plus cigarette smoke induces the excessive generation of reactive oxygen species and free radicals to a greater extent than that from alcohol or cigarette smoke alone. Thus, the combination of cigarette smoking and alcohol drinking might induce the excessive generation of free radicals and cause the marked depletion of serum carotenoid concentrations of regular alcohol drinkers among current smokers than those of non-smokers who have the same alcohol intake.

It is also known that a transforming reaction from pro-vitamin A to retinol is induced by smoking ${ }^{(42)}$. On the other hand, alcohol is known to promote increased oxidation of vitamin A compounds and reduced liver stores ${ }^{(43)}$. It can be postulated that alcohol intake may also accelerate the conversion of pro-vitamin $\mathrm{A}$ to retinol $^{(44)}$. From among the six major serum carotenoids we measured, $\alpha$-carotene, $\beta$-carotene and $\beta$-cryptoxanthin are pro-vitamin A. These three carotenoids are converted to retinol in the body. Therefore, the serum concentrations of $\alpha$-carotene, $\beta$-carotene and $\beta$-cryptoxanthin might be more easily influenced by cigarette smoking and alcohol drinking than lycopene.

In the present study, serum lutein and zeaxanthin concentrations were not influenced not only by alcohol drinking but also by cigarette smoking. We have no clear explanation for these inconsistencies with previous results ${ }^{(25,27-29,31)}$. We concluded that lutein and zeaxanthin might be difficult to be exposed to oxidative stress or that the differences among the six serum carotenoids observed occurred by chance. One possible explanation is that the differences in the associations of the six serum carotenoids with cigarette smoking and alcohol drinking might be attributed to the polar characteristics of each carotenoid. It is conceivable that the tissue distribution and localisation in the cell membranes of carotenoids differ in each carotenoid. Especially, the chemical structure of a carotenoid may determine its localisation in a cell membrane. Hydrocarbon carotenoids, such as lycopene, $\alpha$-carotene and 
$\beta$-carotene, are located within the hydrophobic membrane core with multiple orientations, whereas xanthophylls, such as lutein and zeaxanthin, have a more rigid membrane-spanning orientation ${ }^{(45)}$. Therefore, we believe that the antioxidant defence system by carotenoids against lipid peroxidation in a cell membrane depends on the polar characteristics of each carotenoid. Alternatively, as another one possible explanation for the differences in the associations of the six serum carotenoids with cigarette smoking and alcohol drinking, we consider the differences of carotenoid distribution in lipoproteins. Lipid-soluble carotenoids are carried by lipoproteins from the liver into the blood circulation. Hydrocarbon carotenoids, such as lycopene, $\alpha$-carotene and $\beta$-carotene, are mainly found in LDL, whereas xanthophylls are equally found in HDL and $\mathrm{LDL}^{(46)}$. Recent studies indicate that oxidised LDL increases in smokers and/or heavy alcohol drinkers ${ }^{(47,48)}$. Therefore, it is conceivable that the plasma clearance rate of carotenoids might be influenced by oxidised LDL induced by cigarette smoking and alcohol drinking.

The present study had some limitations. First, we could not evaluate the association of blood concentrations of vitamins $\mathrm{C}$ and $\mathrm{E}$ with cigarette smoking and alcohol drinking. It would be necessary to measure the blood concentrations of vitamins $\mathrm{C}$ and $\mathrm{E}$ in order to examine the associations of these antioxidant vitamin concentrations with these lifestyle factors. Second, the data obtained here consisted of cross-sectional analyses. Therefore, only limited inferences can be made regarding temporality and causation. Third, in the present study, the sample size of current smokers was not particularly large. Therefore, it was impossible to examine the quantitative effects of cigarette smoking on the serum carotenoid concentration. Further studies on a large scale will be required. Fourth, seasonal influence was not considered in the present study, but it could be important to consider this influence in future studies. Last, our findings might be specific to middle-aged Japanese. Further studies in other races and/or regions will be required.

\section{Acknowledgements}

The present study was supported by a grant from the Ministry of Agriculture, Forestry, and Fisheries (MAFF) for a food research project titled 'Integrated Research on Safety and Physiological Function of Food' and a grant from the Council for the advancement of Fruit Tree Science. We are grateful to the participants in our survey and to the staff of the health examination programme for residents of the town of Mikkabi, Shizuoka, Japan. We are also grateful to the staff of the Seirei Preventive Health Care Centre (Shizuoka, Japan).

M. S. was responsible for study design, data collection, and data management and carried out the data analysis and wrote the manuscript. M. N. was responsible for study design, data collection, and data management and assisted in manuscript preparation. K. O., Y. I., H. M., F. A., H. S. and M. Y. were involved in the data collection and assisted in manuscript preparation. All the authors provided suggestions during the preparation of the manuscript and approved the final version submitted for publication.

None of the authors had any personal or financial conflict of interest.

\section{References}

1. Gutteridge JM (1994) Biological origin of free radicals, and mechanisms of antioxidant protection. Chem Biol Interact 91, $133-140$.

2. Rock CL, Jacob RA \& Bowen PE (1996) Update on the biological characteristics of the antioxidant micronutrients: vitamin $\mathrm{C}$, vitamin E, and the carotenoids. J Am Diet Assoc 96, 693-702.

3. World Health Organization (2003) Diet, Nutrition and the Prevention of Chronic Diseases. Joint WHO/FAO Expert Consultation. WHO Technical Report Series no. 916. Geneva: WHO.

4. Bazzano LA, He J, Ogden LG, et al. (2002) Fruit and vegetable intake and risk of cardiovascular disease in US adults: the First National Health and Nutrition Examination Survey Epidemiologic Follow-up Study. Am J Clin Nutr 76, 93-99.

5. Montonen J, Jarvinen R, Heliovaara M, et al. (2005) Food consumption and the incidence of type II diabetes mellitus. Eur J Clin Nutr 59, 441-448.

6. Stanner SA, Hughes J, Kelly CN, et al. (2004) A review of the epidemiological evidence for the 'antioxidant hypothesis'. Public Health Nutr 7, 407-422.

7. Knekt P, Ritz J, Pereira MA, et al. (2004) Antioxidant vitamins and coronary heart disease risk: a pooled analysis of 9 cohorts. Am J Clin Nutr 80, 1508-1520.

8. Ford ES, Will JC, Bowman BA, et al. (1999) Diabetes mellitus and serum carotenoids: findings from the Third National Health and Nutrition Examination Survey. Am J Epidemiol 149, $168-176$.

9. Church DF \& Pryor WA (1985) Free-radical chemistry of cigarette smoke and its toxicological implications. Environ Health Perspect 64, 111-126.

10. Pryor WA \& Stone K (1993) Oxidants in cigarette smoke. Radicals, hydrogen peroxide, peroxynitrate, and peroxynitrite. Ann N Y Acad Sci 686, 12-28.

11. Griendling KK \& FitzGerald GA (2003) Oxidative stress and cardiovascular injury: Part II: animal and human studies. Circulation 108, 2034-2040.

12. Bruno RS \& Traber MG (2006) Vitamin E biokinetics, oxidative stress and cigarette smoking. Pathophysiology 13, 143-149.

13. Chávez J, Cano C, Souki A, et al. (2007) Effect of cigarette smoking on the oxidant/antioxidant balance in healthy subjects. Am J Ther 14, 189-193.

14. Walsh K \& Alexander G (2000) Alcoholic liver disease. Postgrad Med J 76, 280-286.

15. Zima T, Fialova L, Mestek O, et al. (2001) Oxidative stress, metabolism of ethanol and alcohol-related diseases. J Biomed Sci 8, 59-70.

16. Koch OR, Pani G, Borrello S, et al. (2004) Oxidative stress and antioxidant defenses in ethanol-induced cell injury. Mol Aspects Med 25, 191-198.

17. Lieber CS (1993) Herman Award Lecture, 1993: a personal perspective on alcohol, nutrition, and the liver. Am J Clin Nutr 58, 430-442.

18. Eiserich JP, van der Vliet A, Handelman GJ, et al. (1995) Dietary antioxidants and cigarette smoke-induced biomolecular damage: a complex interaction. Am J Clin Nutr 62, $1490 \mathrm{~S}-1500 \mathrm{~S}$.

19. Russell-Briefel R, Bates MW \& Kuller LH (1985) The relationship of plasma carotenoids to health and biochemical factors in middle-aged men. Am J Epidemiol 122, 741-749.

20. Aoki K, Ito Y, Sasaki R, et al. (1987) Smoking, alcohol drinking and serum carotenoids levels. Jpn J Cancer Res 78, 1049-1056.

21. Stryker WS, Kaplan LA, Stein EA, et al. (1988) The relation of diet, cigarette smoking, and alcohol consumption to plasma $\beta$-carotene and $\alpha$-tocopherol levels. Am J Epidemiol 127, 283-296. 
22. Ito Y, Minohara M, Otani M, et al. (1989) Effects of alcohol drinking and cigarette smoking on serum $\alpha$ - and $\beta$-carotene concentrations in healthy adults. Jpn J Hyg 44, 607-614.

23. Tanabe N, Toyoshima H, Hayashi S, et al. (1992) Effects of smoking and drinking habits and vitamin $\mathrm{A}$ intake on serum concentration of $\beta$-carotene and retinol. Jpn J Hyg 47, 679-687.

24. Simonetti P, Cestaro B, Porrini M, et al. (1993) Effect of alcohol intake on lipids and fat-soluble vitamins in blood. Minerva Med 84, 447-452.

25. Tsubono Y, Tsugane S \& Gey KF (1996) Differential effects of cigarette smoking and alcohol consumption on the plasma levels of carotenoids in middle-aged Japanese men. Jpn J Cancer Res 87, 563-569.

26. Fukao A, Tsubono Y, Kawamura M, et al. (1996) The independent association of smoking and drinking with serum $\beta$-carotene levels among males in Miyagi, Japan. Int J Epidemiol 25, 300-306.

27. Brady WE, Mares-Perlman JA, Bowen P, et al. (1996) Human serum carotenoid concentrations are related to physiologic and lifestyle factors. J Nutr 126, 129-137.

28. Rock CL, Thornquist MD, Kristal AR, et al. (1999) Demographic, dietary and lifestyle factors differentially explain variability in serum carotenoids and fat-soluble vitamins: baseline results from the sentinel site of the Olestra Post-Marketing Surveillance Study. J Nutr 129, 855-864.

29. Dietrich M, Block G, Norkus EP, et al. (2003) Smoking and exposure to environmental tobacco smoke decrease some plasma antioxidants and increase $\gamma$-tocopherol in vivo after adjustment for dietary antioxidant intakes. Am J Clin Nutr 77, $160-166$.

30. Galan P, Viteri FE, Bertrais S, et al. (2005) Serum concentrations of $\beta$-carotene, vitamins $\mathrm{C}$ and $\mathrm{E}$, zinc and selenium are influenced by sex, age, diet, smoking status, alcohol consumption and corpulence in a general French adult population. Eur J Clin Nutr 59, 1181-1190.

31. Gabriel HE, Liu Z, Crott JW, et al. (2006) A comparison of carotenoids, retinoids, and tocopherols in the serum and buccal mucosa of chronic cigarette smokers versus nonsmokers. Cancer Epidemiol Biomarkers Prev 15, 993-999.

32. Bieri JG, Brown ED \& Smith JC (1985) Determination of individual carotenoids in human plasma by high performance liquid chromatography. J Liq Chromatogr 8, 473-484.

33. Sugiura M, Nakamura M, Ikoma Y, et al. (2005) High serum carotenoids are inversely associated with serum $\gamma$-glutamyltransferase in alcohol drinkers within normal liver function. J Epidemiol 15, 180-186.
34. Wakai K, Egami I, Kato K, et al. (1999) A simple food frequency questionnaire for Japanese diet - Part I. Development of the questionnaire, and reproducibility and validity for food groups. J Epidemiol 9, 216-226.

35. Egami I, Wakai K, Kato K, et al. (1999) A simple food frequency questionnaire for Japanese diet - Part II. Reproducibility and validity for nutrient intakes. $J$ Epidemiol 9, 227-234

36. Science and Technology Agency (1983) Standard Tables of Food Composition in Japan (in Japanese), 4th ed. Tokyo: Printing Bureau, Ministry of Finance.

37. Yano M, Kato M, Ikoma Y, et al. (2005) Quantitation of carotenoids in raw and processed fruits in Japan. Food Sci Technol Res 11, 13-18.

38. Aizawa K \& Inakuma T (2007) Quantitation of carotenoids in commonly consumed vegetables in Japan. Food Sci Technol Res 13, 247-252.

39. Sandhir R, Subramanian S \& Koul A (2003) Long-term smoking and ethanol exposure accentuates oxidative stress in hearts of mice. Cardiovasc Toxicol 3, 135-140.

40. Husain K, Scott BR, Reddy SK, et al. (2001) Chronic ethanol and nicotine interaction on rat tissue antioxidant defense system. Alcohol 25, 89-97.

41. Hartwig W, Werner J, Ryschich E, et al. (2000) Cigarette smoke enhances ethanol-induced pancreatic injury. Pancreas 21, $272-278$

42. Goodman DS (1984) Overview of current knowledge of metabolism of vitamin A and carotenoids. J Natl Cancer Inst 73, $1375-1379$.

43. Leo MA \& Lieber CS (1982) Hepatic vitamin A depletion in alcoholic liver injury. $N$ Engl J Med 307, 597-601.

44. During A \& Harrison EH (2004) Intestinal absorption and metabolism of carotenoids: insights from cell culture. Arch Biochem Biophys 430, 77-88.

45. Chaudière J \& Ferrari-Iliou R (1999) Intracellular antioxidants: from chemical to biochemical mechanisms. Food Chem Toxicol 37, 949-962.

46. Romanchik JE, Morel DW \& Harrison EH (1995) Distributions of carotenoids and $\alpha$-tocopherol among lipoproteins do not change when human plasma is incubated in vitro. $J$ Nutr $\mathbf{1 2 5}$, 2610-2617.

47. Alho H, Sillanaukee P, Kalela A, et al. (2004) Alcohol misuse increases serum antibodies to oxidized LDL and C-reactive protein. Alcohol Alcohol 39, 312-315.

48. Yamaguchi Y, Haginaka J, Morimoto S, et al. (2005) Facilitated nitration and oxidation of LDL in cigarette smokers. Eur J Clin Invest 35, 186-193. 